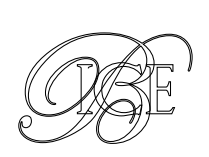

\title{
LA NEGOCIACIÓN DEL TRATADO DE LIBRE COMERCIO DE AMÉRICA DEL NORTE (TLCAN)
}

Este trabajo trata sobre el TLCAN y la negociación emprendida para su renovación entre los países socios: Estados Unidos, México y Canadá. Las posturas son en principio altamente equidistantes, ya que EEUU parte de una posición intransigente en defensa de sus intereses abanderados por el «America first», mientras que México se basa en una negociación que suponga «ganar, ganar, ganar» para los tres socios. Y Canadá, más próximo y alineado con México, defiende la equidad del acuerdo. En lo que están de acuerdo los tres socios es en su actualización y modernización, por lo cual se habla de un Tratado 2.0.

Palabras clave: política económica internacional, acuerdo comercial regional, aranceles, libre comercio.

Clasificación JEL: F13, F14.

\section{Introducción}

Los Tratados de Libre Comercio (TLC) forman parte del puzle organizado de la economía y son una de las piezas más importantes. Consisten en un acuerdo comercial regional o bilateral para ampliar el mercado de bienes y servicios entre los países participantes. Generalmente implica la eliminación o rebaja sustancial de los aranceles para los bienes entre las partes y acuerdos en materia de servicios. Normalmente se rigen por las reglas de la Organización Mundial del Comercio (OMC) o por mutuo acuerdo entre los países.

\footnotetext{
* Consultor Estratégico Iberoamericano. Profesor del Instituto Universitario de Investigación en Estudios Latinoamericanos de la Universidad de Alcalá. Profesor del Instituto de Estudios Bursátiles y de la Escuela Diplomática del Ministerio de Asuntos Exteriores y Cooperación. Gobierno de España.

Versión de marzo de 2018.
}

Un TLC no necesariamente conlleva una integración económica, social y política regional, como es el caso de la Unión Europea, la Comunidad Andina de Naciones, el Mercosur y la Comunidad Sudamericana de Naciones. Si bien estos se crearon para fomentar el intercambio comercial, también incluyeron cláusulas de política fiscal y presupuestaria, así como el movimiento de personas y organismos políticos comunes, elementos ausentes en un TLC.

Nada nuevo desde Adam Smith, que sentó las bases de la economía clásica, la ventaja comparativa y el libre comercio en su magna obra Investigación sobre la naturaleza y causas de la riqueza de las naciones $(1776)^{1}, \triangleright$

Véase la excelente edición que realiza la Fundación del Instituto de Crédito Oficial a cargo de Luis Perdices de Blas en colaboración con John Reeder. Colección Clásicos de Economía: Investigación sobre la naturaleza y causas de la riqueza de las naciones. Madrid, 2010. 
libro donde criticó abiertamente la doctrina económica predominante de la época: «el mercantilismo", cuya máxima era: exportar lo máximo posible, importar lo mínimo posible. Aunque Smith al mismo tiempo afirmó en varias ocasiones que «el comercio interno (es) probablemente el sector comercial más importante en el que con el mismo capital se puede conseguir el incremento más potente de la riqueza del país y el mayor impacto en el empleo».

Como dice Paul Samuelson (premio Nobel de Economía, 1970): «Los economistas discuten todo el tiempo, solo parece que se ponen de acuerdo en relación al libre comercio». Mientras que Paul Krugman (premio Nobel de Economía, 2008) opina que si existiera algo similar a un credo de la economía, con total seguridad incluiría estas normas: "Comprendo el principio de la ventaja comparativa y apoyo el libre comercio». Jagdish Bhagwati, reconocido paladín del libre comercio, afirma: «No me canso de enseñar a mis estudiantes que la tarea de defender constantemente con conocimientos científicos el libre comercio es una obligación».Y $Y$ a pesar de tener numerosos aliados en la lucha, «todavía no forman el ejército que puedo dirigir y que necesitamos». Al mismo tiempo lamenta el hecho de que «el libre comercio rara vez goza de credibilidad entre la población en general».

Así que 212 años después de Smith, en 1988, los Gobiernos de Canadá y Estados Unidos acordaron un «acuerdo de libre comercio» que entró en vigor el 1 de enero de 1989. La finalidad era eliminar todos los aranceles sobre el comercio entre ambos países para 1998. A esto le siguieron las negociaciones con México, para establecer un tratado entre los tres países. Tras cuatro años, concluyeron en agosto de 1992 con un principio de acuerdo y al siguiente año los respectivos Gobiernos de
EEUU, Canadá y México ratificaron el Tratado de Libre Comercio de América del Norte (TLCAN), que se convirtió en ley y entró en vigor el 1 de enero de 1994 (Recuadro 1).

Como resultado, el TLCAN, alumbró un proyecto regional de alta trascendencia, «no solo por el hecho de que vinculaba por primera vez a dos países avanzados con uno en desarro\|lo» en un área de libre comercio sobre bases de plena igualdad y reciprocidad, lo que ya de por sí resultaba un hito sin precedente, sino porque estableció el acuerdo regional más ambicioso, después de la Unión Europea, para la liberalización e integración del comercio y la inversión entre países miembros del GATT -hoy Organización Mundial del Comercio, OMC- (Vega, 2010).

Actualmente, transcurridos veinticuatro años desde la entrada en vigor del TLCAN, los tres países en la negociación, que se inició oficialmente el 16 de agosto de 2017, preconizan su modernización o actualización por resultar tan conveniente como necesaria, para promover que las respectivas economías consigan un mayor grado de integración y competitividad, por encontrarse más «expuestas» a la competencia internacional, con China a la cabeza. Desde esta perspectiva, se estimula la búsqueda de nuevas oportunidades e iniciativas de negocios y de reasignación de los factores de producción de la manera más eficiente.

Las principales áreas que México, Estados Unidos y Canadá desean incorporar al TLCAN son: competitividad, coherencia regulatoria y facilitación del comercio, incluido el electrónico (cuya importancia en el comercio global ha avanzado sin que las reglamentaciones sigan su ritmo $)^{2}$, y los puntos en los que se desea $D$

2 La OMC ha acordado no imponer derechos de aduana a las transacciones electrónicas, pero no se ha progresado en establecer reglas específicas. 


\section{RECUADRO 1 \\ CONTENIDOS Y PUNTOS MÁS IMPORTANTES DEL TLCAN}

- Entrada en vigor el 1 de enero de 1994.

- En 2004, supresión de los aranceles sobre el 99 por 100 de los bienes comerciales entre México, Canadá y Estados Unidos.

- Supresión de la mayor parte de las restricciones sobre la inversión extranjera directa, si bien con un tratamiento especial (de protección) a los sectores mexicanos de energía, ferrocarril, y aéreo, al sector de radiocomunicaciones estadounidense y al sector de la cultura canadiense.

- Aplicación de estándares ecológicos nacionales, siempre que tengan una base científica. Se califica como inapropiado rebajar estos estándares para atraer inversiones.

- Formación de dos comisiones con el poder de imponer multas y cancelar privilegios comerciales si no se respetan los estándares ecológicos o la legislación sobre seguridad e higiene, salarios mínimos y trabajo infantil.

- Queda excluida la inmigración, excepto para los profesionales a los que se les facilite el tráfico interfronterizo.

- Cualquiera de los países miembros puede salirse del tratado con un preaviso de seis meses invocando el artículo 2205.

- Se podrán establecer paneles para la solución de diferencias con especialistas independientes (similar al GATT, 1994).

- Protección de los derechos de propiedad intelectual.

- Se prevé una cláusula de salvaguardia en el caso de que las importaciones puedan dañar la producción nacional.

- EEUU y México establecerán un Banco de Desarrollo de América del Norte*.
- Mercados públicos. Estarán abiertos en diez años. México se reserva algunos contratos exclusivamente para empresas mexicanas.

- Automóvil. Los aranceles se eliminarán en diez años y las cuotas a la importación vigentes en México se retirarán en ese periodo de tiempo. Los automóviles que contengan el 62,5 por 100 de contenido local quedarán libres de aranceles.

- Energía. Pemex continuará con el monopolio nacional de petróleo.

- Agricultura. La mayoría de los aranceles entre EEUU y México se eliminaran inmediatamente. Algunos productos como el azúcar, el maíz, las hortalizas y las frutas, con aranceles del 6 por 100 , quedan excluidos completamente tras un periodo transitorio de quince años.

- Textil. Los aranceles de los tres países quedarán eliminados a lo largo de un periodo transitorio de diez años.

- Servicios financieros. Eliminación de la mayoría de las barreras de servicios entre los países. EEUU y Canadá, desde 2000, tendrán acceso sin límites al mercado mexicano. México eliminará progresivamente los obstáculos a las inversiones en el sector hasta el año 2007, cuando se eliminarán totalmente.

- Transporte pesado. Libre circulación de los camiones en el año 2000.

- Condiciones de trabajo. Los países están obligados al cumplimiento de leyes o normas de seguridad, salud y salario mínimo.

- Ayudas compensatorias. EEUU destinará 90 millones de dólares a lo largo de los primeros 18 meses en ayuda de los trabajadores que tengan problemas con su trabajo, por la puesta en vigor del tratado.

\footnotetext{
* El Banco de Desarrollo de América del Norte es una institución financiera binacional capitalizada y administrada igualmente por los gobiernos federales de Estados Unidos y de México, con el propósito de financiar los proyectos medioambientales certificados por la Comisión de Cooperación Ecológica Fronteriza.
}

Fuente: elaboración propia. 
concentrar son: i) inversión, ii) servicios financieros, iii) telecomunicaciones, iv) propiedad intelectual, v) medioambiente, vi) regulación laboral, vii) energía, viii) empresas estatales, ix) reglas de origen ${ }^{3}$ y $x$ ) solución de controversias/diferencias —un punto este que en todos los tratados comerciales resulta «conflictivo»-, y todo ello con el objetivo «central» de cerrar la gran brecha del déficit comercial que año tras año crece con México, según las siguientes cifras:

1. EEUU, en 2017, obtuvo un déficit comercial total de 566.000 millones de dólares (importó 2,9 billones de dólares y exportó 2,34 billones). Representa el nivel más alto en nueve años y un incremento del 12,1 por 100 respecto a 2016.

2. EEUU tuvo un saldo negativo de bienes situado en 810.000 millones de dólares y logró un superávit en servicios de 244.000 millones.

3. EEUU, con México, históricamente mantiene un déficit comercial desfavorable. En 2017 alcanzó 71.060 millones de dólares (el mayor déficit desde 2007, con la entonces cifra récord de 69.311 millones de dólares). En un año superó el registrado por Japón (68.800 millones), Alemania (64.200 millones) y Canadá (17.500 millones).

4. EEUU mantiene el mayor déficit comercial con China, que en 2017 se situó en 375.200 millones de dólares. China, con diferencia, es el mayor socio comercial de EEUU, y el déficit comercial es cinco veces superior al de México.

\footnotetext{
3 Las reglas de origen tienen el propósito de asegurar que la liberalización del comercio beneficie exclusivamente a las empresas que producen en el territorio de los países miembros del tratado para impedir que terceros utilicen a una de las partes como plataforma para introducir sus productos.
}

Teniendo en cuenta que el TLCAN es un factor de certidumbre en la integración de América de Norte, es indispensable mantener y promover disposiciones que contribuyan a hacer más previsibles las operaciones de comercio y las inversiones. En ello juegan un rol crucial los mecanismos de solución de controversias que, a lo largo de todos estos años, han probado su efectividad como un instrumento que permite hacer más predecible el comercio y la inversión regional, además de incorporar los elementos más recientes de transparencia y participación de otros actores relevantes.

\section{Sobre la negociación del TLCAN}

Las posturas ante la negociación del Tratado de Libre Comercio de América del Norte son equidistantes y parten de visiones e intereses distintos.

El presidente de EEUU, Donald J. Trump, califica al TLCAN como el «peor acuerdo de comercio» aprobado por su país, culpable directo de terribles déficits comerciales, pérdidas de empleos, producción manufacturera y cierre de fábricas. Su postura negociadora se fundamenta en sus ideas «proteccionistas», regidas por el «America first» (América primero), que trasmite «el final de una era basada en el libre comercio como eje de la globalización». Ahora bien, el hecho concreto es que no ha ordenado la retirada de EEUU del TLCAN, como sí lo hizo del Acuerdo Transpacífico de Cooperación Económica (TPP), o su oposición a no firmar el Transatlantic Trade and Investment Partnership (Área de Libre Comercio Transatlántico, TTIP). Lo que sí tiene como línea roja Trump es su propia «imprevisibilidad».

Por parte del presidente de México, Enrique Peña Nieto, la postura negociadora adoptada $\triangleright$ 
desde el principio es constructiva e integradora, apostando —como Canadá- por el «libre comercio", reconociendo que el TLCAN ha sido un revulsivo para la economía mexicana y que constituye una pieza clave para su crecimiento, modernización e integración regional, que en gran parte se produce mediante las cadenas de suministro (también llamadas redes de producción internacional o cadenas globales de valor) que a su vez resultan claves para la competitividad de EEUU en manufacturas, productos electrónicos y de consumo. Aunque ha fijado su línea roja en la no construcción del «muro» que reivindica el presidente Trump, para México constituye un asunto interno de Estados Unidos, aunque lo considere un gesto «inamistoso».

En cuanto al primer ministro de Canadá, Justin Trudeau, su postura parte de su convencimiento y visión positiva del tratado para su país y los respectivos socios. Desde el principio estableció un diálogo fluido con ambos presidentes, y especialmente en poder convencer a Trump para hacerle ver lo bueno que ha resultado y resultará aún más el nuevo acuerdo. Pero también ha marcado una línea roja. Se trata de que EEUU retire su propuesta de eliminar del tratado el capítulo 19, que determina el mecanismo para la revisión y resolución de controversias sobre subsidios o cuotas compensatorias y dumping, permitiendo que los países soliciten el establecimiento de paneles binacionales independientes cuando sus exportadores o productores sientan que son víctimas de decisiones comerciales desleales por algún otro socio. Estos paneles emiten decisiones vinculantes para resolver la disputa. La propuesta de EEUU es que las controversias se diriman exclusivamente en EEUU, lo cual supone un enorme retroceso que dificultaría la negociación. Canadá ya tiene un precedente, retiró a su jefe negociador de las conversaciones de 1987 sobre el Tratado Canadá-EEUU.

Las negociaciones, una vez concluida la sexta ronda (Montreal, 23-29 de enero de 2018), se presentan cada vez más arduas y complejas. Solo hay que esperar para saber si finalmente la cuerda de los negociadores se romperá o aguantará el leitmotiv de EEUU, "América primero», y se cumple lo que México desea como resultado final: que los tres países ganen, lo que significa ser más competitivos regional e internacionalmente, incorporando los beneficios para conseguir una mejor y mayor cohesión económica y social.

Robert Lighthizer, representante comercial de Estados Unidos, estima que las negociaciones deberían compensar el daño hecho a los trabajadores del sector manufacturero de su país y que el tratado no debe beneficiar a unos a expensas de otros. Christya Freeland, ministra de Asuntos Exteriores de Canadá, considera que su país está en la negociación para fortalecer aún más los lazos comerciales trilaterales. Ildefonso Guajardo, secretario de Economía de México, encuentra muy positivas las negociaciones, las cuales han sido altamente beneficiosas. Sin embargo, sobre los tres puntos más controvertidos, solución de controversias, reglas de origen y déficit comercial, no se han logrado avances concretos.

Ya desde la cuarta ronda (Washington, 1017 de octubre de 2017) se esperaba que estos tres puntos se abordasen, pero ni siquiera se trataron. Tampoco la propuesta más añorada por Trump, reducir el déficit comercial con México, no resultará nada fácil, porque no reconoce que representa solo una pequeña parte de su déficit global. Como tampoco reconoce que los acuerdos de libre comercio no tienen como cometido eliminar los déficits comerciales. La función de un TLC debe ser, y $\triangleright$ 
tradicionalmente lo es, aumentar el comercio y estimular la economía en general, pero no reducir el déficit comercial.

Concluida la quinta ronda (México DF, 1721 de noviembre de 2017), se realizó, como en las anteriores, un comunicado conjunto por parte de los tres países informando que se reunieron 30 grupos negociadores que avanzaron en varios capítulos, pero sin cerrar ninguno. De manera que se llegó a final de año sin cumplirse el plazo establecido para concluir la negociación. $Y$ visto que no se avanzaba al ritmo planeado, se acordó extender las negociaciones hasta finales de marzo de 2018, para lo cual habrá que pisar el acelerador si se desea verdaderamente cerrar la negociación exitosamente.

Robert Lighthizer se muestra preocupado porque, finalizada la quinta ronda, no observa «avances significativos». Le preocupa que Canadá y México no estén dialogando seriamente sobre las cláusulas que llevan a un nuevo equilibrio en el acuerdo: «De no alcanzar este nuevo equilibrio, no llegaremos a un resultado satisfactorio». Considera que un TLCAN reequilibrado y actualizado promoverá mayor prosperidad para los trabajadores, agricultores, ganaderos y empresarios estadounidenses, y fortalecerá a América del Norte en su totalidad. Lo que entiende Lighthizer por un TLCAN reequilibrado es la actualización de las reglas de origen como propone EEUU. Esto es pasar en la industria automotriz del 62,5 por 100 actual al 85 por 100 propuesto por su país, con la pretensión de asegurarse que, al menos, el 50 por 100 de ese total fuese fabricado en EEUU.

Por su parte, Ildefonso Guajardo ratificó la importancia de México como socio comercial estratégico para EEUU y para la competitividad regional del TLCAN, y esto con la importancia de otorgar certidumbre a las inversiones, a la vez que reiteraba la posición constructiva y positiva de México para lograr un acuerdo que beneficie a los tres países.

Iniciado el nuevo año, se llevó a cabo la sexta ronda (Montreal, 23-29 de enero de 2018). El tiempo apremia y las resoluciones también, pues hasta la fecha, lo que se dice avanzar, no se ha avanzado.

Nuevamente, Robert Lighthizer calificó las propuestas de México y Canadá como inadecuadas y, en algunos casos, como contrarias a las demandas estadounidenses: «Esta sexta ronda fue un paso hacia adelante, pero estamos progresando lentamente. Debemos a nuestros ciudadanos, que están viviendo una situación de incertidumbre, un progreso mucho más rápido». En el mismo sentido se expresó Ildefonso Guajardo: «Los tres países se encuentran en un mejor momento negociador y el progreso realizado hasta ahora se encamina a poder aterrizar los temas y concluir el proceso".

Hasta ahora, los negociadores han logrado concluir solo tres capítulos: pymes, competencia y anticorrupción. Adicionalmente, concluyeron el anexo sectorial sobre tecnologías de la información y comunicación, y se encuentran por terminar las negociaciones en los anexos de productos farmacéuticos y químicos. Únicamente tres capítulos, de los veintiocho que se están negociando, presentan un avance importante y se cerraron en la séptima ronda (México DF, del 26 de febrero al 6 de marzo de 2018). Otros sobre telecomunicaciones, comercio digital y medidas sanitarias y fitosanitarias han avanzado bastante y se confía que puedan concluirse en la siguiente ronda; mientras que aduanas y facilitación del comercio incluirán nuevas normas vinculantes y están redactadas a un nivel superior del logrado en el marco de la Organización Mundial del Comercio y mucho más alto que en el TPP. 
Entre los datos adversos resalta el choque entre EEUU con Canadá. Chrystia Freeland, ministra de Relaciones Exteriores de Canadá, defiende que su propuesta sobre las reglas de origen busca beneficiar a la región intentando no arruinar las cadenas logísticas transfronterizas y su impulso a la competitividad. De manera que Canadá para nada desea debilitar la competitividad de Estados Unidos, sino más bien fortalecerla. No opina así Lighthizer, quien señaló que la propuesta en materia de reglas de origen llevaría a una menor generación de empleo en los tres países: «Nos parece que las reglas del sector automotriz como se presentaron por parte de Canadá pueden conducir a menos contenido regional del que tenemos ahora, lo cual significaría menos trabajo en EEUU, Canadá y posiblemente México, así que tendríamos lo opuesto de lo que estamos tratando de lograr».

A todo esto, recuérdese que el TLCAN adquiere una alta relevancia histórica, porque nunca antes dos naciones desarrolladas habían firmado ese tipo de acuerdo con un país en vías de desarrollo en un área de libre comercio y sobre bases de plena reciprocidad. Contenía, por tanto, una reversión extraordinaria de la sabiduría convencional de la teoría de la integración económica que se adoptó desde 1950, que asumía que las áreas de libre comercio entre los países desarrollados y en desarrollo no serían del interés de los últimos.

También el TLCAN estableció el acuerdo regional más ambicioso, después de la Unión Europea, para la liberalización e integración del comercio y la inversión entre países miembros del GATT, estableciendo de esta manera lo que por entonces resultaba ser la segunda área de libre comercio más grande del mundo, siendo la primera la Unión Europea. Los objetivos del tratado se limitaban a una zona de libre comercio en bienes y servicios, sirviendo como modelo para posteriores acuerdos de Estados Unidos como los que firmó con Chile (2003), América Central (2004), Perú (2006) y Colombia (2008).

Así que los negociadores están descubriendo la lentitud. Cuando dieron comienzo en agosto de 2017, confiaban en que finalizarían en diciembre de 2017. Seguramente no tuvieron en cuenta que las anteriores negociaciones del TLCAN tardaron cuatro años, desde 1988 hasta 1992, y los Gobiernos de EEUU, Canadá y México lo ratificaron en 1993, convirtiéndose en ley el 1 de enero de 1994.

\section{La posible retirada de Estados Unidos}

Sobre la posibilidad de que la negociación no llegase a su fin, las consecuencias serían altamente perjudiciales para México, pero también para Estados Unidos, y de manera especial para la industria manufacturera, electrónica y agrícola, que pagarían hasta el 35 por 100 al aplicarse los aranceles de la nación más favorecida, estipulados en el marco de la OMC, mientras que Estados Unidos aplicaría a México un promedio del 3 por 100.

Se hace evidente, pues, que EEUU no puede ignorar hechos tan relevantes como que México adquiere más productos estadounidenses que las tres potencias mundiales juntas: Alemania, Japón y Reino Unido. Además, es con mucho el primer socio comercial de Estados Unidos en mercancías, el segundo destino comercial después de Canadá y el tercer socio comercial después de Canadá y China. Razón de peso, al menos, para que las negociaciones lleguen a su término.

Ahora bien, si EEUU decide retirarse de la negociación, dando por concluido el tratado, $\triangleright$ 
México cuenta con un «Plan B» de diversificación geográfica de las exportaciones, que encuentra su opción más próxima en América del Sur, adonde solo exporta el 4,3 por 100 del total. Este nivel le permite una gran capacidad de crecimiento a través de sus socios en la Alianza del Pacífico (Chile, Colombia y Perú) y Mercosur (Argentina y Brasil), pero también con países tan notables como Japón y China, su segundo socio comercial, que valora negociar un TLC, y regiones como la Unión Europea, con quien tiene muy avanzadas las negociaciones del nuevo Tratado de Libre Comercio Unión Europea-México (TLCUEM). Esta perspectiva toma fuerza, incluso si la negociación del tratado termina bien, así aprenderá a mirar comercialmente más allá de EEUU.

México, si bien debe contrarrestar la excesiva dependencia comercial de EEUU, no debe impedir maximizar sus exportaciones, atraer nuevas inversiones y aumentar su integración por medio de las cadenas de suministro. Mirar más allá de EEUU, para no estar pendiente cada cuatro años de quién es el nuevo inquilino de la Casa Blanca, debe ser su gran meta económica y comercial.

Aunque la meta es altamente aconsejable, sin embargo no resulta nada fácil de llevar a cabo. $Y$ es que las fuertes interrelaciones entre ambos países, y de la manera que interactúan, dan cuenta que este es un mundo muy difícil de reemplazar, casi imposible. A lo que hay que sumar el profundo conocimiento que las empresas de ambos países tienen de ambos mercados.

El otro «Plan B» se encuadra en lo político. En el inmediato horizonte, y cada vez más, toma cuerpo la idea de que las negociaciones se extiendan más allá de la última ronda prevista para finales de marzo de 2018. Esto introduce más incertidumbre a los ciudadanos, y a las empresas, cuyos planes de producción, expansión e inversión se ven afectados. $Y$ esta idea se abre paso sencillamente porque los tres países tienen en su calendario político diversas citas electorales. Elecciones generales en México (julio), legislativas de medio término en Estados Unidos (noviembre) y locales en Canadá (Toronto y Quebec, entre otras ciudades). Por tanto, la idea de que las negociaciones se extenderán hasta 2019 gana terreno, lo que indudablemente no pueden ignorar o pasar por alto las intensas relaciones que mantienen EEUU y México que marcan las negociaciones ${ }^{4}$.

\section{Conclusiones}

Muy posiblemente, la clave del resultado final de la negociación se encuentra en el «nivel» de aceptación que EEUU obtenga de sus socios sobre su «América primero».

Más allá de las controversias y los desencuentros en la negociación, sería deseable que se tuviese en cuenta que la modernización del tratado representa un punto de encuentro e interés mutuo entre EEUU, México y Canadá. A cada uno la modernización le abre nuevas perspectivas. México es el país que más puede ganar, en tanto en cuanto le brinda la oportunidad de generar un desarrollo más equilibrado. Los desequilibrios entre los estados del Sur y del Norte desde la firma del TLCAN han sido evidentes. La población de los estados del Norte se ha duplicado ampliamente dado su mayor desarrollo. EI PIB per cápita ha crecido un 47 por 100, mientras que en los estados del Sur lo ha hecho el 7 por 100. No solo esto: $\square$

\footnotetext{
4 Para más detalles, véase gráficamente: Panorama comercial de EEUU con el mundo y México. https://fgsaenzfgs.blog/2018/02/08/ panorama-comercial-de-ee-uu-con-el-mundo-y-mexico-analisis-graficol
} 
actualmente los estados del Norte son 2,6 veces más productivos que los del Sur, lo cual agranda las distancias $y$, en consecuencia, ahonda los desequilibrios económicos dado que persiste el desfase de la producción y comercio que se realiza en un 85 por 100 desde los estados del Norte con EEUU.

El propósito de la negociación pasa por conseguir ampliar y mejorar los logros económicos que el TLCAN ha conseguido para México, sin olvidar la cohesión social, cuyos indicadores hablan por sí solos: el 1 por 100 más rico de la población cuenta con el 33 por 100 de la riqueza nacional, en tanto que el 10 por 100 acumula el 66 por 100. Tampoco debe olvidarse la cohesión del sistema productivo, donde solo el 20 por 100 de las pequeñas y medianas empresas exportan sus productos, pese a que representan el 99,8 por 100 del total del sistema productivo y generan el 73 por 100 del empleo formal.

Definitivamente, el nuevo TLCAN debe contribuir con renovados impulsos para que México cuente con una economía fuerte, cohesionada, estable y dinámica y sin muros de ningún tipo, sino con puentes por donde transitar libremente. Para México, avanzar en esta dirección significa contar con nuevas dinámicas que, además, hacen posible una mayor y más efectiva integración con América Latina, donde, como segunda potencia regional, debe desempeñar un papel de primer nivel.

Por todo lo cual, el fortalecimiento de las relaciones económicas y comerciales de México con EEUU continuará siendo una prioridad de su política económica internacional ${ }^{5}$. El tamaño del mercado de EEUU, las amplias interrelaciones creadas y el liderazgo en innovación tecnológica sugieren que seguirá siendo el

5 Conjunto de medidas que asumen los Estados con el propósito de incidir en el entorno económico, incluyendo el flujo de bienes, servicios y capitales de acuerdo con lo que considera como su mejor interés. principal destino de las exportaciones mexicanas y fuente de inversión extranjera directa.

Más aún, sería ampliamente deseable que la modernización del TLCAN prepare a la región para insertarse exitosamente en la nueva geografía económica mundial, la cual está configurando un nuevo patrón de comercio internacional, caracterizado por contar con un número creciente de cadenas de suministro globales o redes de producción global. Por consiguiente, el reto de los tres países debe transformarse en una nueva «oportunidad». Esta se encuentra en saber alumbrar inteligentemente una negociación que promueva una visión competitiva que fomente los intercambios comerciales del futuro, cuyas señales evidentes pasan por la economía digital, donde EEUU hará valer sus potentes y amplios intereses.

En cuanto a la situación de la negociación, se encuentra tal como comenzó en la primera ronda, envuelta por la «incertidumbre», que no ha desaparecido, pues no se han producido avances ni acuerdos significativos al menos hasta la sexta ronda. Sí se han producido desacuerdos relevantes tras las propuestas que EEUU realizó en la cuarta ronda, referidas a las normas de origen, los requisitos de contenido, la eliminación de los mecanismos de resolución de controversias y la introducción de una cláusula de revisión cada cinco años del tratado (muerte súbita). Propuestas que han erosionado la relación EEUU-Canadá, la cual se encuentra en su punto histórico más tenso.

Así, las sucesivas rondas no están resultando precisamente efectivas, sino que están trabando las negociaciones. Razón por la cual, aunque debían concluir a finales de 2017, se decidió continuarlas al menos hasta marzo de 2018. Para entonces, la campaña electoral presidencial de México estaba subida de tono, mientras que las elecciones legislativas de $\square$ 
medio término en Estados Unidos se encontraban lo suficientemente cerca para influir sobre las negociaciones. Canadá, aunque enfrenta comicios municipales, no siente la presión de sus dos socios. No obstante, estos deben tener muy presente que el TLCAN genera más de un billón de dólares anuales en comercio entre los tres países, lo que significa el 40 por 100 del producto interno bruto de Canadá, el 49 por 100 de México y el 5 por 100 de Estados Unidos.

En cuanto a los posibles impactos del nuevo TLCAN sobre las empresas españolas, estas se enfrentan en el mercado mexicano a una competencia similar con la que se encuentran en otros países. No obstante, cuentan con una ventaja comparativa muy importante; la Marca España es bien reconocida, percibida y valorada, ya que la gestión, la tecnología y el conocimiento de nuestras empresas están contribuyendo, y seguirán haciéndolo, al desarrollo, competitividad y modernización de la economía mexicana.

Actualmente, en México operan empresas españolas de todo tamaño, tipo y condición que suman más de 5.800 . España, con una inversión extranjera directa de 40.114.592 millones de euros, mantiene el primer puesto europeo y el segundo mundial, solo por detrás de EEUU. Respecto a México, en las preferencias de las empresas españolas, se sitúa como el segundo destino inversor en América Latina (Brasil es el primero) y el cuarto en el mundo, únicamente superado por EEUU, Reino Unido y Brasil. La IED española se concentra en servicios financieros que incluyen seguros, telecomunicaciones, suministro de energía eléctrica, eólica, solar y gas, infraestructuras, turismo e ingeniería civil.

El ministro de Asuntos Exteriores y Cooperación de España, al reunirse durante su visita oficial a México con los empresarios españoles, les expresó que la situación de las relaciones económicas, comerciales y de inversión entre ambos países las considera muy positivas y en el mejor nivel y sintonía.

Para finalizar algunas preguntas para la reflexión. ¿Qué puede esperarse de la política económica internacional de Estados Unidos sobre el TLCAN? ¿Hasta qué punto la estructura finalmente acordada en el TPP podría emplearse para la modernización del TLCAN? ¿Cuál es el futuro del TLCAN y la integración regional que el nuevo acuerdo impulsa? ¿Los riesgos implícitos y explícitos que conlleva una ruptura de la negociación del TLCAN serán suficientes para evitarlo?

\section{Bibliografía}

[1] CASILDA BÉJAR, R. (2002). La década dorada. Economía e inversiones españolas en América Latina 1990-2000. Servicio de publicaciones de la Universidad de Alcalá. Madrid.

[2] CASILDA BÉJAR, R. (2011). Multinacionales españolas en un mundo global y multipolar. ESIC Business School. Madrid.

[3] CASILDA BÉJAR, R. (2016). «La internacionalización de las empresas españolas en Latinoamérica desde la perspectiva inversora». Papeles de Economía Española, n.․150. FUNCAS. Madrid.

[4] CASILDA BÉJAR, R. (2017). La negociación de México-Estados Unidos del Tratado de Libre Comercio de América del Norte y su posible impacto en las empresas españolas. Documento de Trabajo, oㅡ 89. Fundación Alternativas. Madrid.

[5] CASILDA BÉJAR, R. (2018). La gran apuesta. Globalización y multinacionales españolas en América Latina. Análisis de los protagonistas. Ediciones Granica. Barcelona y Bogotá.

[6] VEGA CÁNOVAS, G. (2010). El tratado de libre comercio en América del Norte. Visión retrospectiva y retos a futuro. Centro de Estudios Internacionales. El Colegio de México. México DF. 